\title{
Propagation Of A Stress Wave Through A Virtual Functionally Graded Foam
}

\author{
Stephen Kiernan ${ }^{\text {a }}$ Liang Cui ${ }^{a}$ Michael D. Gilchrist ${ }^{\text {a,* }}$ \\ ${ }^{a}$ School of Electrical, Electronic $\&$ Mechanical Engineering, University College \\ Dublin, Ireland
}

\begin{abstract}
Stress wave propagation through a Functionally Graded Foam Material (FGFM) is analysed in this paper using the Finite Element Method. A Finite Element model of the Split Hopkinson Pressure Bar (SHPB) is developed to apply realistic boundary conditions to a uniform density foam and is validated against laboratory SHPB tests. Wave propagation through virtual FGFMs with various gradient functions are then considered. The amplitude of the stress wave is found to be shaped by the gradient functions, i.e. the stress can be amplified or diminished following propagation through the FGFMs. The plastic dissipation energy in the specimens is also shaped by the gradient functions. This property of FGFMs provides significant potential for such materials to be used for cushioning structures.
\end{abstract}

Key words:

Stress wave propagation, Functionally Graded Material, Split Hopkinson Pressure Bar

\section{Introduction}

Cellular foams are widely used in energy absorbing applications where it is important to minimise the peak acceleration of the impacting body [1], e.g packaging of fragile goods, protective headgear $[2,3]$ and body garments. This is due to their low volume fraction of solid material and their complex microstructure which allows large degrees of plastic crushing to occur at a fairly constant stress value. This plastic crushing at a constant stress will continue until, depending on their initial density, a densification strain is reached when

* Corresponding author. 
cell walls and struts impinge on one another and further crushing is of the material matrix itself rather than the foam cells. Understanding their dynamic stress-strain behaviour at finite deformations is therefore essential in order to predict their performance as cushioning materials.

Much research has been performed, both experimentally and numerically, on the finite deformation of both polymeric and metallic foams under quasi-static to moderate strain-rate conditions. However, there does not appear to be an obvious consensus in the literature as to the dynamic behaviour of metallic foams w.r.t. strain rate sensitivity. Deshpande and Fleck [4] reported that the plateau stress in closed cell Alulight and open cell Duocel metallic foams are almost strain rate insensitive, up to rates of 5000/s. An open celled AA6101T6 AL foam was investigated by Lankford and Dannemann [5], who reported almost no change in mechanical strength in response to varying strain rates. Peroni [6] also reported strain rate insensitivity for aluminium foam. However, Kanahashi et al [7] have investigated the dynamic strain rate response of open celled SG91A aluminium foam at a rate of 1400/s and reported a strain rate dependence. Dannemann and Lankford Jr [8] reported a strain rate effect of closed cell ALPORAS foam at strain rates between 400 and 2500/s. They noted that strain rate effects were higher for a higher density and attribute this to the kinetics of internal gas flow. Elnasri et al [9] reported a limited rate sensitivity for ALPORAS foam at strain rates up to $1300 \mathrm{~m} / \mathrm{s}$. Zhao and Abdennadher [10] stated that the rate sensitivity of metallic foam is due to inertia effects in dynamic buckling of cell walls, although the foam is made of strain rate insensitive material. Klintworth [11] and Reid and Peng [12] discussed the possibility for the strength increase in cellular structures that, under dynamic conditions, the collapse mechanism of the foam changes from the quasi-static mode to a dynamic mode involving additional stretching of the cell wall that dissipates more energy.

The recently emerging field of Functionally Graded Materials(FGMs) was initially limited to create metal-ceramic composites to combat high thermal gradients in the aerospace industry, but can also be applied to cellular structures. It has already been shown numerically [13] that Functionally Graded Foam Materials (FGFMs) are suitable candidates for providing improved energy absorbing properties over those provided by conventional foams of uniform densities. Bruck [14] has studied FGMs to analyse the effect of a gradient architecture on the mitigation of stress waves reflected from the graded interface. Berezovski [15] extended the study of stress wave propagation from one dimension to two dimensions for metal-ceramic FGMs. The current study examines wave propagation through a virtual FGFM, using a traditional Split Hopkinson Pressure Bar setup to apply meaningful boundary conditions, and to elucidate the general energy absorbing mechanisms of this type of material. Metallic foam, rather than polymeric foam, was used in the current study as the former has higher impedance and is easier to test using the SHPB facility 
in our laboratory.

Manufacturing a FGFM is inherently more complex than a uniform foam due to the varying material properties, however, some authors have reported on technologies to produce such materials. Brother and Dunand [16], for example, created density graded aluminium foams from polyurethane foam precursors using an investment casting method. This was in an effort to improve mass-efficiency in load bearing metallic foams. Matsumoto et al [17] have proposed an alternate and interesting method to producing density graded foams - rather than introducing the gradient at the time of foaming, their method is based on chemical dissolution of a uniform foam. By immersing the uniform foam within a $\mathrm{NaOH}$ bath of controlled $\mathrm{pH}$ and then draining the $\mathrm{NaOH}$ by gravity at a constant rate, they introduced a continuous gradient in exposure time of the solution to the uniform foam - thereby creating a continuous density gradient. Kieback et al [18] also developed a metallurgical process for FGMs which were formed through graded metal powder compacts and followed by melt processing. As described by Kieback et al, manufacturing FGMs in the laboratory scale has reached a considerable level of maturity, however, there will be new challenges including manufacturing processes to mass production and up-scaling, and cost-effectiveness of production processes. The technology to manufacture the functionally graded polymeric foam material is presently being developed in our laboratory and will form the basis of a future publication.

The Split Hopkinson Pressure Bar was designed to capture the dynamic stressstrain response of materials at very high strain rates. A typical arrangement of the SHPB is shown in Fig. 1. A stress pulse, generated by the impact of the striker bar onto the incident bar, travels down the incident bar and interacts with the specimen. Some of this stress pulse is transmitted through the specimen to the transmitter bar, and some of the pulse is reflected back to the incident bar. Strain gauges, mounted on the incident and transmitter bars, record the incident strain, $\varepsilon_{I}(t)$, the transmitted strain, $\varepsilon_{T}(t)$, and the reflected strain, $\varepsilon_{R}(t)$ of these two bars. The stress-strain response, $\sigma_{s}(t)$ and $\varepsilon_{s}(t)$, (and the strain rate of the response, $\left.\dot{\varepsilon}_{s}(t)\right)$ of the specimen can be reconstructed from these strain-time records by applying the well known equations:

$$
\begin{aligned}
\dot{\varepsilon}_{s}(t) & =-\frac{2 C_{0}}{L_{s}} \varepsilon_{R}(t) \\
\varepsilon_{s}(t) & =-\frac{2 C_{0}}{L_{s}} \int_{0}^{t} \varepsilon_{R}(t) d t \\
\sigma_{s}(t) & =E_{b} \frac{A_{b}}{A_{s}} \varepsilon_{T}(t)
\end{aligned}
$$


where $C_{0}$ is the wave speed within the bar, $L_{s}$ is the length/thickness of the specimen, $A_{s}$ and $A_{b}$ are the cross-sectional area of the specimen and the bar, respectively, and $E_{b}$ is Young's modulus of the bar.

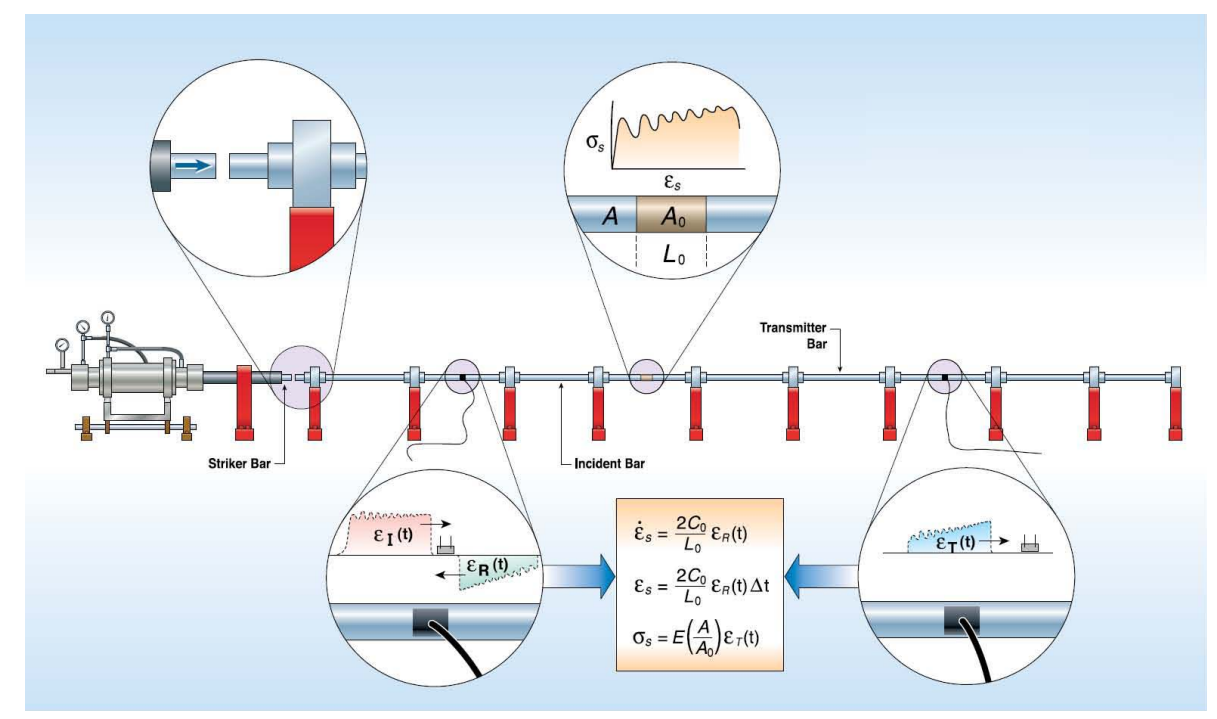

Fig. 1. Schematic illustration of the Split Hopkinson Pressure Bar (SHPB). [19]

The SHPB technique has proved to be extremely versatile and has grown from its original configuration for compression testing to include tension, torsion and fracture testing [20]. It has been used to characterise the dynamic response of a multitude of materials such as soils [21], composites [22], compliant materials [23] and metals [24]. Foams have also been studied using the SHPB technique [25].

Al-Mousawi et al [20] provided a good summary of the limitations of using the Split Hopkinson technique in dynamic testing, including uniaxial stress distribution through the specimen, bar-specimen frictional forces that can cause radial tractions, and the influence of radial dispersion of the pulse wave through the elastic bars. Sawas [26] acknowledged the limitations on high noise-to-signal ratio and low achievable strain when using metal incident and transmitter bars with complaint specimens like rubbers and foams due to the very high impedance mismatch and short rise time of the pulse wave. They introduced an all-polymeric Split Hopkinson Bar system to overcome these limitations. Chen et al [27] noticed the non-homogeneous deformation of the SHPB when using low-impedance specimens, due to the attenuation and slow speed of the wave across the soft material $(\lesssim 500 \mathrm{~m} / \mathrm{s}$ compared to $\gtrsim 5000 \mathrm{~m} / \mathrm{s}$ for metals). In order to achieve near identical force-time histories on both faces of the soft specimen, a thin sample should be used and pulse shaping techniques should be employed. Although these techniques were applied to solid elastomeric materials, the authors point out that they are applicable also to cellular foams. 
In this paper, laboratory SHPB tests are first performed to validate a virtual SHPB setup. The complementary simulations are then carried out using the experimental input parameters. The FE predictions of the incident and reflected waves are quantitatively validated by comparing the laboratory tests with the simulations. Discrepancies between the simulated and experimental transmitted waves are explained by the foam's strain rate sensitivity. In the second half of the paper, a stress wave is propagated through a virtual FGFM to illustrate and analyse the wave propagation characteristics through the FGFM, and compare its response with a uniform foam of equal average density. Much thicker specimens $(\approx 300 \mathrm{~mm})$ than is traditional for SHPB testing are required during these simulations to illustrate the differing response between a uniform and a functionally graded foam. Although using such large thicknesses would immediately invalidate Equations (1) to (3) under experimental conditions, these equations are not required to determine the stress-strain response of the virtual FGFM; stress, strain, and any other values can be determined directly from requested element outputs. In this respect the virtual incident and transmission bars serve only to apply realistic boundary conditions to the FGFM, since they are compared against those from the lab tests, and ensure that a realistic stress wave is used in the model.

\section{Laboratory Tests}

\subsection{Experimental Setup}

In order to achieve comparable impedances between specimen and the pressure bars, and to ensure a transmitted wave with a high signal-to-noise ratio, the incident and transmitter bars used were made from $30 \%$ glass filled nylon 66 with a diameter of $50 \mathrm{~mm}$ and a length of $1000 \mathrm{~mm}$. The bars have a quoted Young's Modulus of $7.5 \mathrm{GPa}$ and density of $1350 \mathrm{~kg} / \mathrm{m}^{3}$, giving a theoretical wave speed of $2357 \mathrm{~m} / \mathrm{s}$. From experiment the wave speed was calculated as $2296 \mathrm{~m} / \mathrm{s}$. The positive $z$-direction was taken to coincide with the bars' longitudinal axes in the direction of the propagating wave. Two projectiles of length $150 \mathrm{~mm}$ and $250 \mathrm{~mm}$ were used during the tests. Each projectile was accelerated using a pneumatic ram powered by pressurised nitrogen. In the current study, a velocity of about $19 \mathrm{~m} / \mathrm{s}$ and $13 \mathrm{~m} / \mathrm{s}$ for the $150 \mathrm{~mm}$ and $250 \mathrm{~mm}$ projectiles, respectively, was achieved using a pressure head of 10 bar respectively. A low velocity was chosen for two reasons: a) minimise the amount of strain and hence dispersion of the stress wave due to the visco-elastic behavior of the nylon bars, b) the laboratory tests serve to validate the numerical model, and so do not require high projectile velocities. A limiting gas pressure was available during the current experiments and so a short projectile length was used out of necessity. A longer projectile would give a longer incident wave but 
would have been of too low amplitude to cause specimen yielding. As no pulse shaping techniques were used in this work, stress uniformity cannot be fully ensured and so the reported laboratory results should only be interpreted as an average stress-strain reading, used to compare against simulation results.

\subsection{Materials}

A single material, ALPORAS ${ }^{\circledR}$ aluminium foam (GLEICH GmbH MetallplattenService, Kaltenkirchen, Germany), was used for all tests and a PTFE spray was used on both incident and transmitter bars to minimise any radial traction of the foam during compression. ALPORAS ${ }^{\circledR}$ foam has a quoted mass density of $250 \mathrm{~kg} / \mathrm{m}^{3}$. The standard deviations (std), and $95 \%$ confidence intervals, (CI) for each specimen length are shown in Table 1. It shows that there is in fact a wide spread in density between samples, which is likely due to random, unfoamed regions, and aluminium agglomerates that will decrease or increase the average density respectively. Young's Modulus of ALPORAS ${ }^{\circledR}$ foam was obtained from quasi-static uniaxial compression to be about $75 \mathrm{MPa}$.

The specimens are $45 \mathrm{~mm}$ in diameter to give an adequate radial representative volume element (RVE), and are of various lengths $l$, as listed in Table 1. Achieving stress uniformity in cellular materials under SHPB conditions is difficult because there is a trade-off between how thin a sample must be to ensure that Equation (3) is valid, and how thick it must be to give enough cells through the thickness for the RVE. Chen et al [28] performed SHPB experiments on polyurethane foams of thickness $1.7 \mathrm{~mm}$, with a cell size of $0.3 \mathrm{~mm}$, giving about 5.5 cells through the thickness. Despande and Fleck [4] carried out similar experiments on Alulight and Duocel foams. They reported specimens having 6 - 8 cells in all specimen dimensions. During our experiments a specimen thickness of $5 \mathrm{~mm}$ was found to be too small to give an accurate RVE of the bulk foam, indeed it was strongly felt that the resulting stress-strain curves obtained represented the response of the individual cells themselves. Specimens of thickness $10 \mathrm{~mm}$, however, yielded between 5 and 7 cells through the thickness and were found to be sufficient.

Fig. 2 shows the representative incident strain and the reflected strain generated by the projectile of length $150 \mathrm{~mm}$ with velocity of $19 \mathrm{~m} / \mathrm{s}$, and the transmitted strain through a specimen of thickness $10 \mathrm{~mm}$, as obtained from the strain gauges. The deduced strain curves and stress curves of the same specimen using Equations (2) and (3) are illustrated in Fig. 3(a) and Fig. 3(b), respectively, and the stress-strain relationships for the $10 \mathrm{~mm}(\dot{\varepsilon} \approx 2000 / \mathrm{s})$ and $20 \mathrm{~mm}(\dot{\varepsilon} \approx 1000 / \mathrm{s})$ specimens are shown and compared to the quasistatic compression curve in Fig. 4. The calculated stress-strain curve exhibits a higher yield stress than the quasi-static compression curve, indicating a strain 
Table 1

Densities, standard deviations and 95\% Confidence Intervals for 5, 10, $20 \mathrm{~mm}$ specimens

\begin{tabular}{|c|c|c|c|}
\hline$l(\mathrm{~mm})$ & $\rho_{\text {avg }}\left(\mathrm{kg} / \mathrm{m}^{3}\right)$ & $\operatorname{std}(\sigma)$ & $95 \%$ CI \\
\hline 5 & 241.0 & 3.25 & $237.0-245.1$ \\
10 & 260.8 & 12.93 & $252.1-269.5$ \\
20 & 270.7 & 26.30 & $253.0-288.4$ \\
All Specimens & 261.2 & 21.18 & $252.8-269.6$ \\
\hline
\end{tabular}

rate sensitivity of the foam; however the elastic modulus does not appear to be strain rate sensitive. In this study, however, our model assumes rate independent plasticity. As strain rate effects are absent from both the uniform and graded foam models and as same strain rate was used for comparison between the uniform and graded foam models, the influence of introducing material gradients into cushioning structures can clearly be quantified. It is intended to implement the strain rate sensitivity into the constitutive model as part of future research.

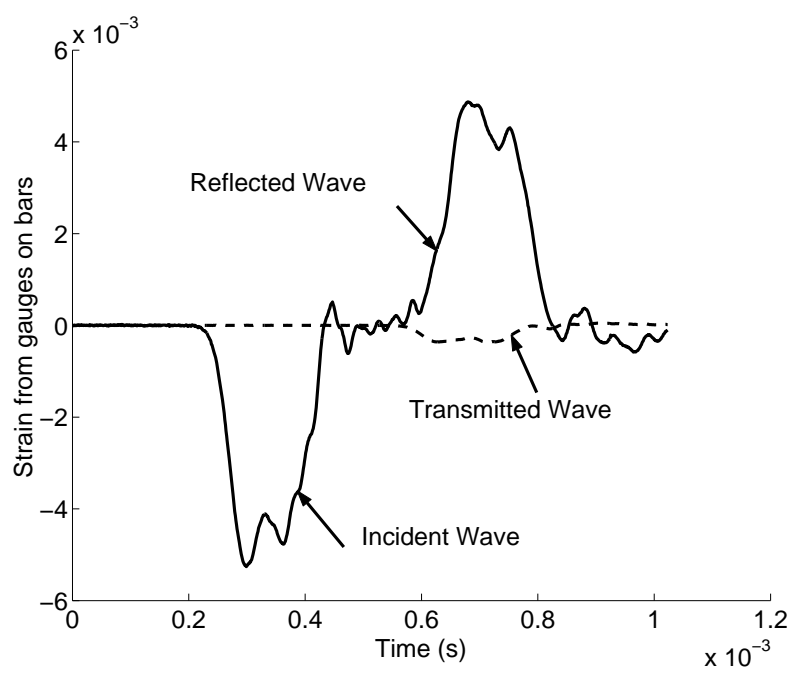

Fig. 2. Representative incident, reflected and transmitted strains as measured from laboratory tests using a $150 \mathrm{~mm}$ projectile.

\subsection{Wave Dispersion}

Although a low projectile velocity was selected during the experiments, wave dispersion was seen to take place as the wave propagated longitudinally down the bar. The testing of low impedance materials such as cellular solids requires that low impedance incident and transmission bars be used to ensure a significant transmission wave is recorded and can be easily discerned from any obvious noise in the system. For such cases viscoelastic bars made from 


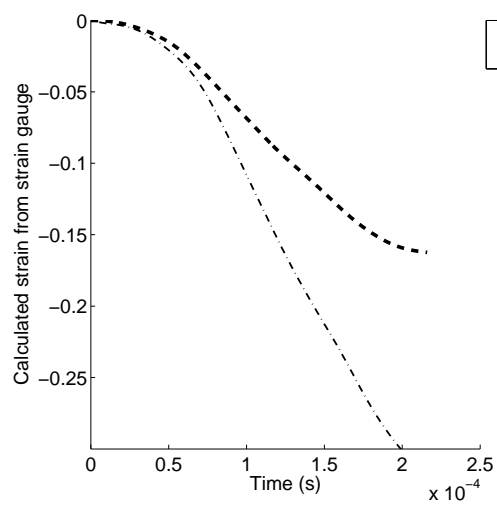

(a) Strain curves

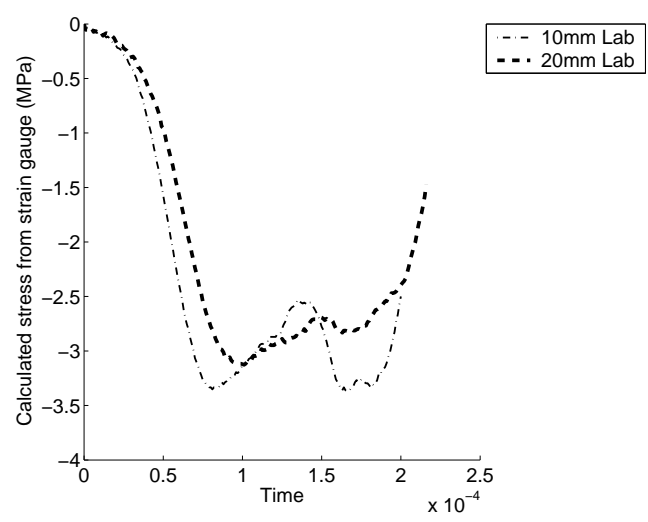

(b) Stress curves

Fig. 3. The deduced strain curves and stress curves from the strain gauges $(150 \mathrm{~mm}$ projectile). (a) Strain curves, (b) Stress Curves

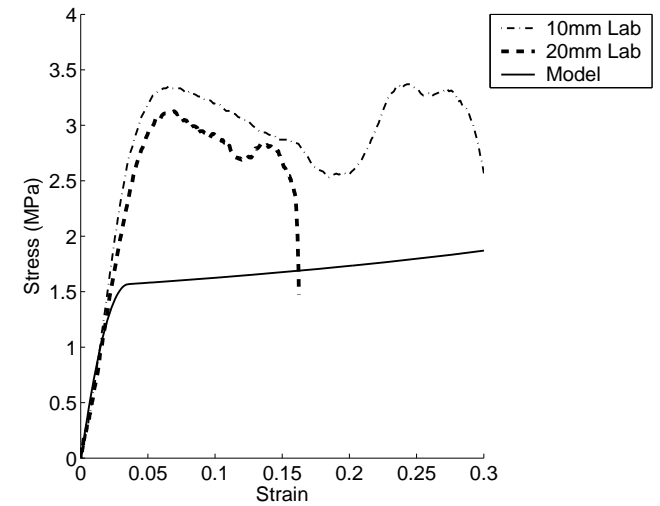

(a) $150 \mathrm{~mm}$ projectile

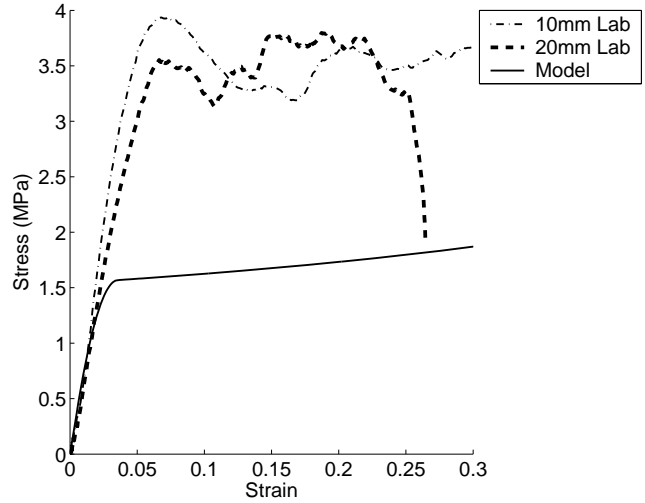

(b) $250 \mathrm{~mm}$ projectile

Fig. 4. The stress-strain curve obtained from the SHPB tests. (a) $150 \mathrm{~mm}$ projectile (b) $250 \mathrm{~mm}$ projectile

nylon or PMMA are suitable options as there are currently few known low impedance pure elastic materials [29]. However due to the viscoelastic nature of these materials, different frequency components of the incident, reflected, and transmission waves will behave differently to each other, i.e. higher frequencies will have a higher phase velocity and will be attenuated quicker than lower frequency components of the waves. This will lead to a change in the wave shape and amplitude as it propagates from one point in the bar to another. Therefore, it is necessary to shift each wave from the point at which it is measured to the specimen-bar interfaces. This was done by transferring the signal (recorded at a position $z_{1}$ ) into the frequency domain using a discrete Fourier transform, processing it according to the work of [30] and [31], and reconstructing the signal back into the time domain at a new position $z_{2}$. 
Figure 5 shows the same wave (input/output) measured at two strain gauges $0.373 \mathrm{~m}$ apart along with the predicted wave at the second strain gauge when a $150 \mathrm{~mm}$ and $250 \mathrm{~mm}$ bullet is used. From the simulations very little change in the wave shape took place in the elastic pressure bars, indicating that wave dispersion was only due to material effects rather than the bar's geometry.

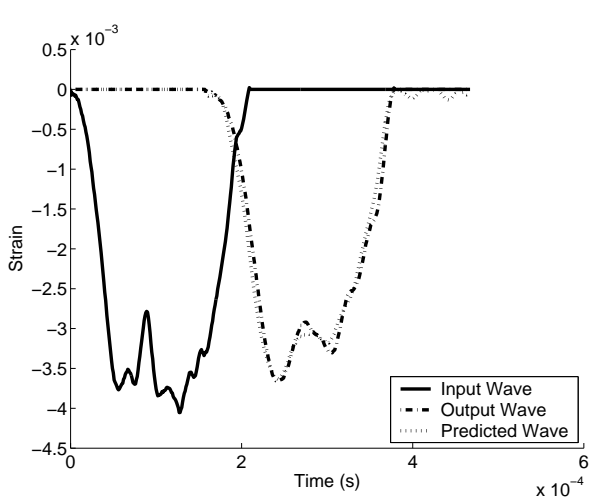

(a) $150 \mathrm{~mm}$

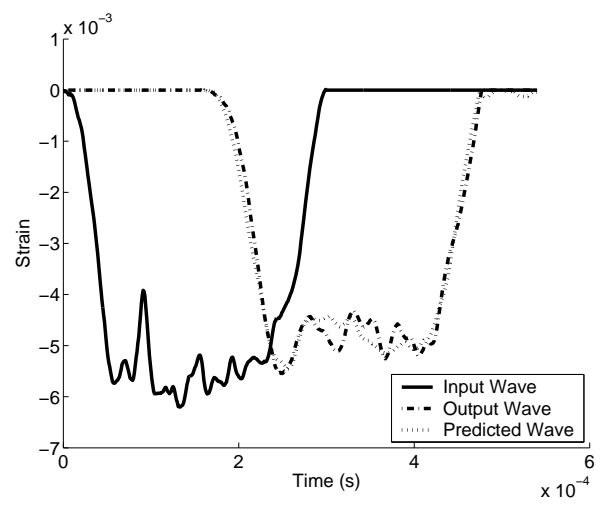

(b) $250 \mathrm{~mm}$

Fig. 5. Change in wave shape over $0.373 \mathrm{~m}$ and predicted wave shape for $150 \mathrm{~mm}$ and $250 \mathrm{~mm}$ projectiles. (a) $150 \mathrm{~mm}$ (b) $250 \mathrm{~mm}$

\section{FEM simulations}

\subsection{Constitutive model for FGFM}

In modelling the uniform foam (and FGFM), the actual topology of the foam (cell, struts, etc) were not modelled and so, from a finite element perspective, the virtual foam cells could be assumed to be infinitely small. The F.E. package ABAQUS allows one to describe the dynamic response of a crushable foam plasticity model by inputting a uniaxial $\sigma-\varepsilon$ curve to calibrate the model provided. The yield surface of the crushable foam is an ellipse in the meridional $(p-q)$ stress plane, as shown in Fig. 6. The evolution of the yield surface follows the volumetric hardening rule, for which the point on the yield ellipse that represents hydrostatic tension loading is fixed and the evolution of the yield surface is driven by the volumetric compacting plastic strain. The shape of the yield surface is determined by the initial yield stress in uniaxial compression, $\sigma_{c}^{0}$, the initial yield stress in hydrostatic compression, $p_{c}^{0}$ (the initial value of $p_{c}$ ), and the yield strength in hydrostatic tension, $p_{t}$. The values of the yield stress in uniaxial compression as a function of the plastic strain can be calculated from the uni-axial compression stress-strain curve obtained from the constitutive stress-strain relationship. 


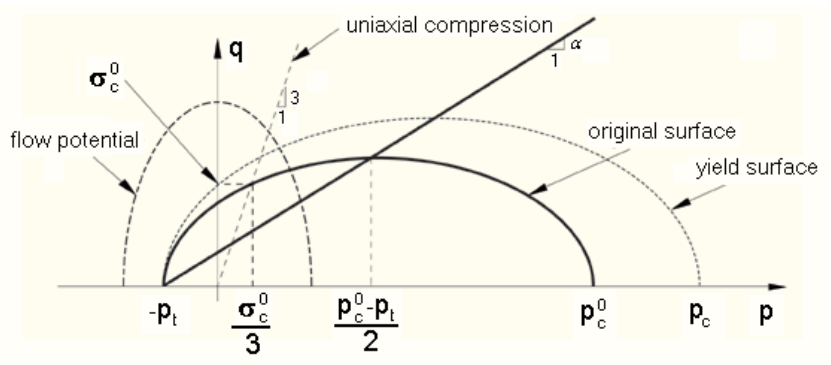

Fig. 6. Crushable foam model with volumetric hardening: yield surface and flow potential in the $p-q$ stress plane [32]

Modelling of the FGFM is achieved by approximating a specimen with a continuous variation in material properties as many discrete, finely meshed element layers though the thickness, with a unique $\sigma-\varepsilon$ curve associated with each layer. As described in the following constitutive model, the foam relative density is the controlling parameter in describing the shape of each $\sigma-\varepsilon$ curve. By varying this parameter in an incremental manner, one can generate multiple $\sigma-\varepsilon$ curves and calibrate the ABAQUS crushable foam model for a range of foam densities. Each calibrated crushable foam model for a given density may then be assigned to a given element layer through the specimen thickness, creating a quasi-graded cellular constitutive response.

The constitutive model describing $\sigma(\varepsilon)$ for each curve was borrowed from Schraad and Harlow [33], describing disordered cellular materials under uniaxial compression according to various stochastic material representations. This model was first applied to the crushable model by Cui et al [13]. The stress-strain relation of the foam was represented by a tri-linear function. With the assumption that the Poisson's ratio for low-density foam is approximately zero, the tangent stiffness $E$ of the foam under uni-axial compression is found to be a function of its solid-volume fraction and the axial strain. That is

$$
E\left(\varepsilon^{n}\right)=A\left(\varepsilon^{n}\right) E_{s}\left[\phi\left(\varepsilon^{n}\right)\right]^{2}
$$

where $\varepsilon^{n}$ is the nominal axial strain (length change per unit undeformed length) and in the range of $(-1,0), A\left(\varepsilon^{n}\right)$ is a stiffness related parameter varied with the axial strain, $E_{s}$ is the tangent stiffness of the parent solid material used to make the cellular material, and $\phi\left(\varepsilon^{n}\right)$ is the relative density, or the solid-volume fraction. As $\varepsilon^{n}=0, A(0)=A_{0}, \phi(0)=\phi_{0}$, and $E(0)=E_{0}$. During the compression, $\phi\left(\varepsilon^{n}\right)$ can be expressed as (with zero Poisson's ratio)

$$
\phi\left(\varepsilon^{n}\right)=\frac{\left(\phi_{0}\right)}{1+\varepsilon^{n}}=\frac{\rho_{0}}{\rho_{s}} \frac{1}{1+\varepsilon^{n}}
$$

where $\rho_{0}$ is the initial density before compression and $\rho_{s}$ is the density of the parent solid material. The parameter $A\left(\varepsilon^{n}\right)$ defines the geometry of the stress- 
strain curve. For axial strains lower than the yield value, $\varepsilon_{1}$, it shows a linear elastic response with a tangent stiffness equal to $E_{0}$. As the axial strain is increased over the yield value, the plateau stress only increases slightly with the steadily increasing axial strain, yielding a smaller tangent stiffness, $E_{1}$. As the axial strain increases further to higher than a densification strain, $\varepsilon_{2}$, the cells are crushed entirely and the cell walls start to contact each other. The stiffness in this stage increases sharply and finally approaches the stiffness of the parent solid material as the axial strain approaches $100 \%$.

As described by Schraad and Harlow [33], the transition between the three stages is over a small range of strain rather than instantaneously, due to the imperfectly homogeneous or identical cellular structure of the foam. Assuming that the imperfection of the cellular structure is distributed randomly, the transition between the linear elastic stage and the plateau stage occurs over a small range of $2 \Delta \varepsilon_{1}$, while the transition between the plateau stage and the densification stage occurs over a small range of $2 \Delta \varepsilon_{2}$. The geometric parameter, $A\left(\varepsilon^{n}\right)$, for the foam can then be expressed as

$$
A\left(\varepsilon^{n}\right)=\left\{\begin{array}{lc}
A_{0}, & \varepsilon_{1}+\Delta \varepsilon_{1} \leq \varepsilon^{n} \leq 0 \\
\frac{\left(A_{0}-A_{1}\right) \varepsilon^{n}-A_{0}\left(\varepsilon_{1}-\Delta \varepsilon_{1}\right)+A_{1}\left(\varepsilon_{1}+\Delta \varepsilon_{1}\right)}{2 \Delta \varepsilon_{1}}, & \varepsilon_{1}-\Delta \varepsilon_{1} \leq \varepsilon^{n} \leq \varepsilon_{1}+\Delta \varepsilon_{1} \\
A_{1}, & \varepsilon_{2}+\Delta \varepsilon_{2} \leq \varepsilon^{n} \leq \varepsilon_{1}-\Delta \varepsilon_{1} \\
\frac{\left(A_{1}-1\right) \varepsilon^{n}-A_{1}\left(\varepsilon_{2}-\Delta \varepsilon_{2}\right)+\left(\varepsilon_{2}+\Delta \varepsilon_{2}\right)}{2 \Delta \varepsilon_{2}}, & \varepsilon_{2}-\Delta \varepsilon_{2} \leq \varepsilon^{n} \leq \varepsilon_{2}+\Delta \varepsilon_{2} \\
1, & -1 \leq \varepsilon^{n} \leq \varepsilon_{2}+\Delta \varepsilon_{2}
\end{array}\right.
$$

where $A_{0}$ and $A_{1}$ can be obtained from Equations (4) and (5) as

$$
\begin{aligned}
& A_{0}=\frac{E_{0}}{E_{s} \phi_{0}^{2}}=\frac{E_{0} \rho_{s}^{2}}{E_{s} \rho_{0}^{2}} \\
& A_{1}=\frac{E_{1}}{E_{s} \phi\left(\varepsilon_{1}\right)^{2}}=\frac{E_{1} \rho_{s}^{2}}{E_{s} \rho_{0}^{2}}\left(1+\varepsilon_{1}\right)^{2}
\end{aligned}
$$

To determine the parameters in the functions of the constitutive model for a specified type of foam, a series of quasi-static uni-axial compression tests were performed on the ALPORAS ${ }^{\circledR}$ foam. The assumption of a vanishing Poisson's ratio for ALPORAS ${ }^{\circledR}$ foam is not strictly correct although uni-axial compression testing up to $80 \%$ strain revealed lateral straining to be only 4$5 \%$. Experimental SHPB testing resulted in maximum strains of about $30 \%$. A summary of the static test results is shown in Fig. 7. For solid Aluminium, $\rho_{s}=2700 \mathrm{~kg} / \mathrm{m}^{3}$ and $E_{s}=80 \mathrm{GPa}$. Based on the results of the experimental compression tests, the parameters in the model can be determined. The 
stress-strain curves obtained from the constitutive formula with the determined parameters are also illustrated in Fig. 7. The constitutive model was found to quantitatively match the results of the experimental tests. From this, stress-strain curves for virtual specimens of higher arbitrary densities were extrapolated and used during the numerical simulations in order to analyse a suitably wide range of densities, as shown in Fig. 8.

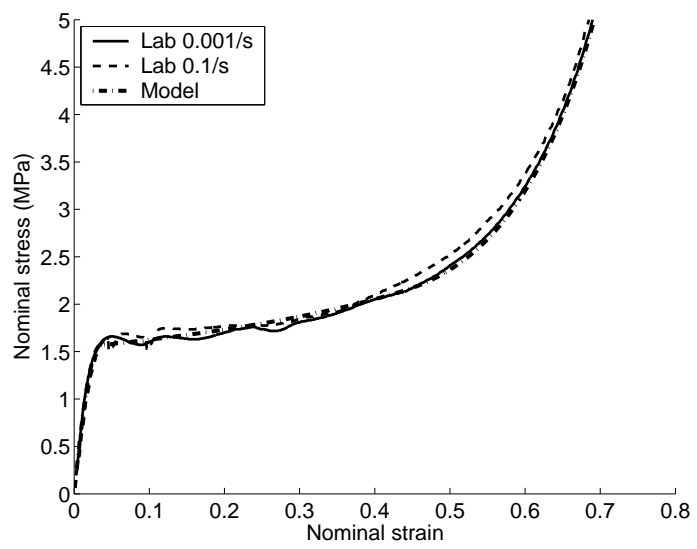

Fig. 7. Quasi-static compression stress-strain curves of the Aluminium foam.

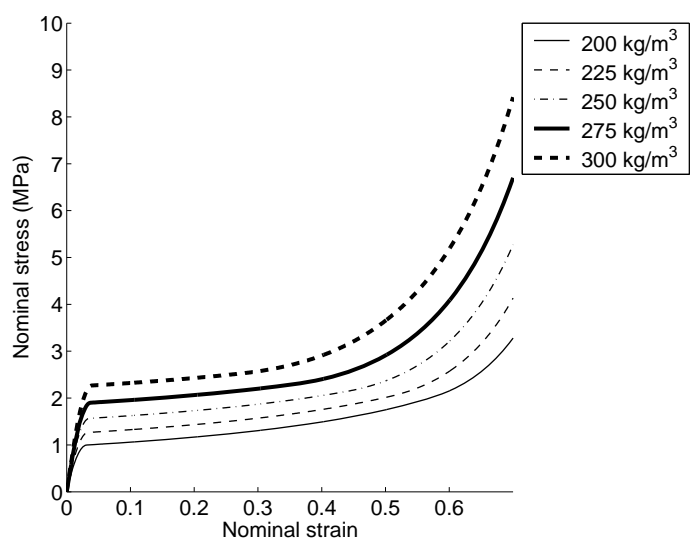

Fig. 8. Virtual stress-strain curves for various foam densities.

In order to describe the hypothetical constitutive response of the functionally graded foam proposed in this paper, the model specimen is meshed finely through the thickness direction, as shown in Fig. 9. A unique $\sigma-\varepsilon$ curve is associated with each element layer. By varying $\sigma(\varepsilon)$ incrementally, and according to a chosen monotonic gradient function, from one element layer to the next, a quasi-gradient FGFM constitutive response could be described. In current study, gradient functions of logarithmic, square root, linear, quadratic, and cubic, as shown in Fig. 10, are used. To perform a parallel comparison, the average density of each specimen is targeted to be the same as that of the uniform foam specimen, $250 \mathrm{~kg} / \mathrm{m}^{3}$. To achieve the same average density the upper and lower limits for the graded density range increases as the gradient changes from convex (logarithmic, square root) to concave (quadratic, and cubic). The density parameters for the FGFM specimens are listed in Table 2. 


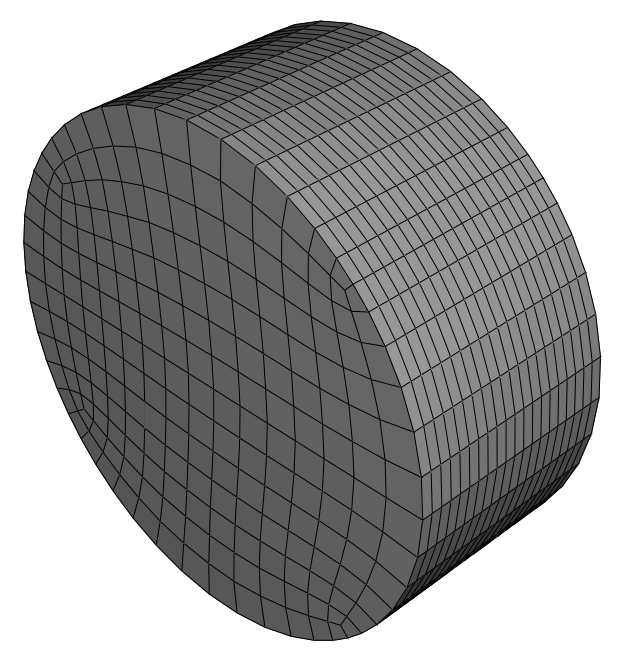

Fig. 9. Mesh configuration of foam specimen (thickness $=20 \mathrm{~mm}$ )

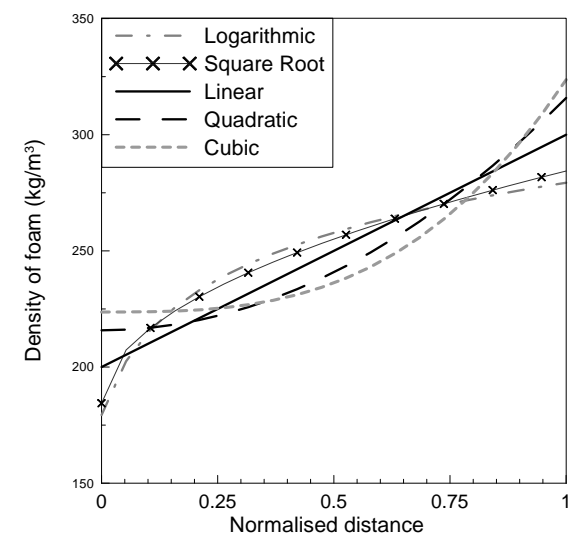

(a) Increasing density

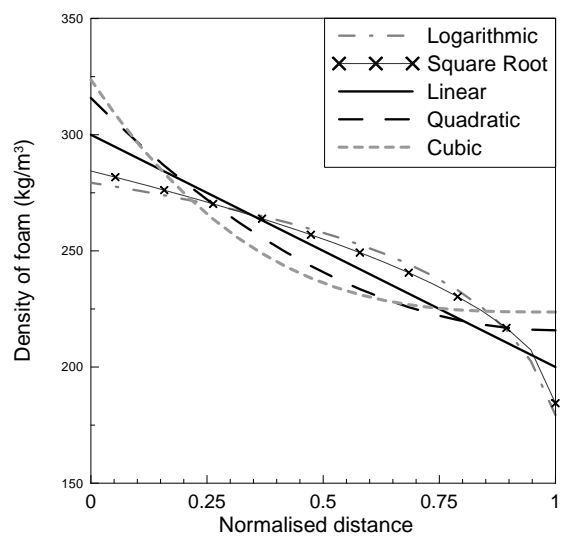

(b) Decreasing density

Fig. 10. Variation in density versus normalised distance to the incident surface $\left(\Delta \rho=100 \mathrm{~kg} / \mathrm{m}^{3}\right)$. (a) Increasing density (b) Decreasing density

\subsection{Description of FE model}

The FEM simulations of the Split-Hopkinson Pressure Bar were performed using the software package ABAQUS [32]. Material parameters measured from the laboratory tests define the constitutive response of the model. Material descriptions of the bars and projectiles are defined as elastic media with the same dimension, density and stiffness as those in the laboratory tests. The foam specimen is modelled as a crushable foam as described in Section 3.1.

All assembly parts are modelled as three-dimensional 8-node linear brick elements using reduced integration with hourglass control (C3D8R). The bars 
Table 2

Density parameters for the FGFM specimens

\begin{tabular}{|l|c|c|c|}
\hline \multirow{2}{*}{ Gradients } & \multicolumn{3}{|c|}{ Density Range $\left(\mathrm{kg} / \mathrm{m}^{3}\right)$} \\
\cline { 2 - 4 } & $\Delta \rho=100$ & $\Delta \rho=50$ & $\Delta \rho=20$ \\
\hline Uniform & 250 & 250 & 250 \\
\hline Logarithmic & $179.34-279.34$ & & \\
\hline Square Root & $184.39-284.39$ & & \\
\hline Linear & $200-300$ & $225-275$ & $240-260$ \\
\hline Quadratic & $215.79-315.79$ & & \\
\hline Cubic & $223.68-323.68$ & & \\
\hline
\end{tabular}

and projectiles have 214 elements in its cross-sectional area. The bars have 180 elements through its thickness. A mesh sensitivity analysis of the incident and transmitter bars using 75, 100 and 150 elements along their lengths showed no significant change in the strain wave profiles. The $150 \mathrm{~mm}$ projectile and $250 \mathrm{~mm}$ projectile have 25 and 50 elements through its thickness, respectively. The foam specimen has 20 elements through its thickness and 214 elements in its cross-sectional area. An explicit central-difference time integration rule is used to simulate the dynamic impact behaviour. The maximum stable time increment is given by

$$
\Delta t=\frac{L_{\min }}{C_{d}}
$$

where $L_{\min }$ is the smallest element dimension in the mesh, $C_{d}$ is the wave propagation speed. The stable time step is chosen and updated automatically by ABAQUS throughout the solution.

The incident velocity of the $150 \mathrm{~mm}$ projectile is $20 \mathrm{~m} / \mathrm{s}$ and the incident velocity of the $250 \mathrm{~mm}$ projectile is $10 \mathrm{~m} / \mathrm{s}$ in the simulations. All the surfaces in contacts are defined as frictionless surface. No gravitational force is included for any part and projectile, specimen and bars are constrained to only move in the z-direction. An initial velocity boundary condition is also applied to the projectile. Strain gauges are defined on the virtual incident and transmitter bars to compare with the laboratory test strain curves and to validate the FE models.

\subsection{Simulation Results - Uniform Specimen}

Comparisons of the incident and reflected strains between the laboratory tests and the FE simulations are illustrated in Fig. 11(a) and match each other 
quantitatively. Comparison of the transmitted strains between the laboratory tests and the FE simulations is also illustrated in Fig. 11(b), however the transmitted strains are lower in the FE simulations than those in the laboratory tests due to the aforementioned strain rate sensitivity of the physical foam.

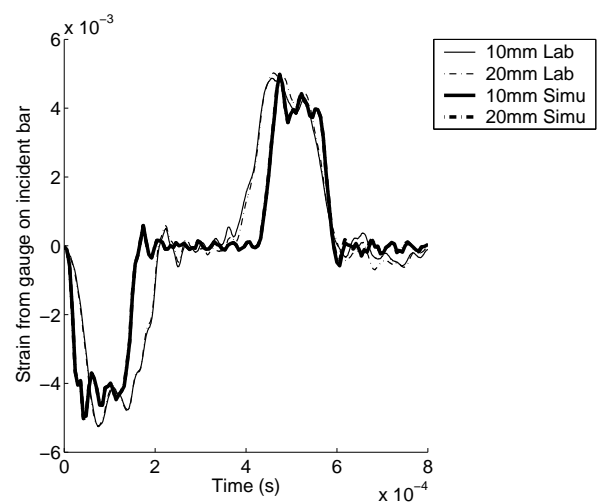

(a) Incident and reflected strains

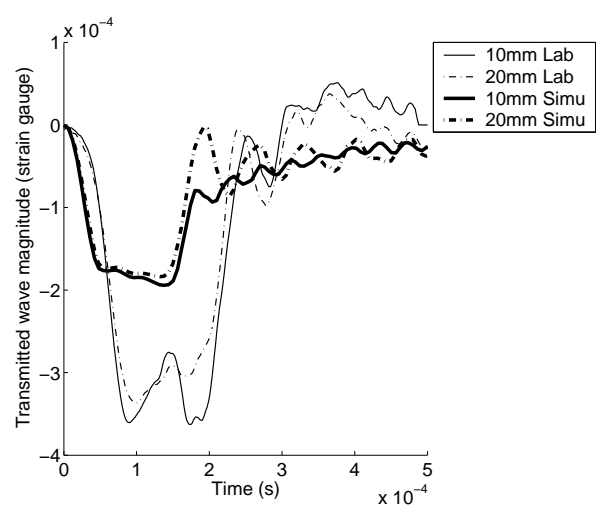

(b) Transmitted strains

Fig. 11. Comparison of the strain gauge readings between the laboratory tests and the FEM simulations. (a) Incident and reflected strains (b) Transmitted strains

The evolution of stress in 10 selected layers from the incident surface to the distal surface of the uniform specimens (thickness $=10 \mathrm{~mm}$ ) is illustrated in Fig. 12. The stress curves for different layers almost coincide except for the peak values, indicating that a state of dynamic stress equilibrium exists in the $10 \mathrm{~mm}$ virtual specimens. The stress and strain responses of the specimen can be calculated from the strain curves from the strain gauges. The average stress and strain response of the whole specimen can also be output directly from the FEM simulations. These two sets of curves are compared in Fig. 13(a) and Fig. 13(b). Stress and strain measurements from the model strain gauges and those measured directly from the $10 \mathrm{~mm}$ thick virtual foam are similar but deviate more as the thickness increases to $20 \mathrm{~mm}$. This should be expected, as Equations (2) and (3) become less valid for increasing thickness. The calculated stress-strain curves from this data coincide with the input stress-stain model curve except for some minor fluctuations, as shown in Fig. 14.

By comparing the stress and strain response of the specimen in the FEM model (Figs. 13(a) and 13(b)) and in the laboratory tests (Figs. 3(a) and 3(b)) with various specimen thickness, and noting the lack of a strain rate definition in the model of Schraad and Harlow [33], the FEM Model is judged to be qualitatively validated. The SHPB model can now be applied more generally to the functionally graded foam by providing appropriate boundary conditions to set up an initial stress wave without the need to rely on Equations (1) to (3) to calculate the constitutive response. 


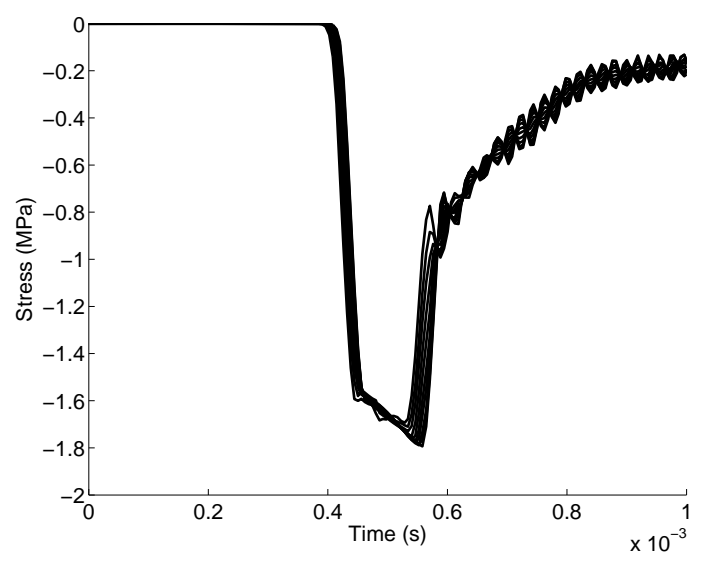

Fig. 12. Evolution of stress in 10 selected layers in the uniform specimen of thickness $10 \mathrm{~mm}$.

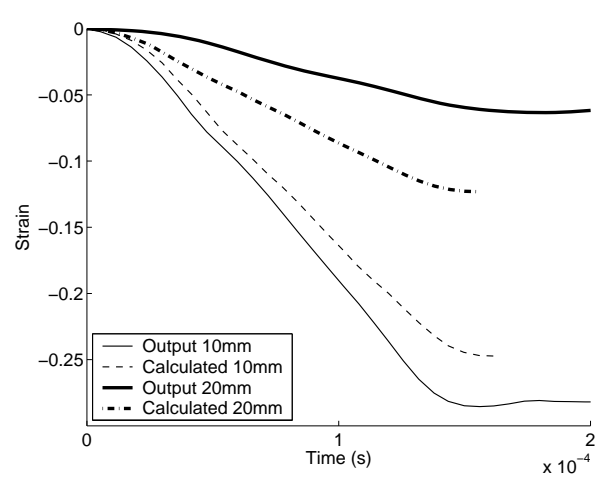

(a) Strain curves

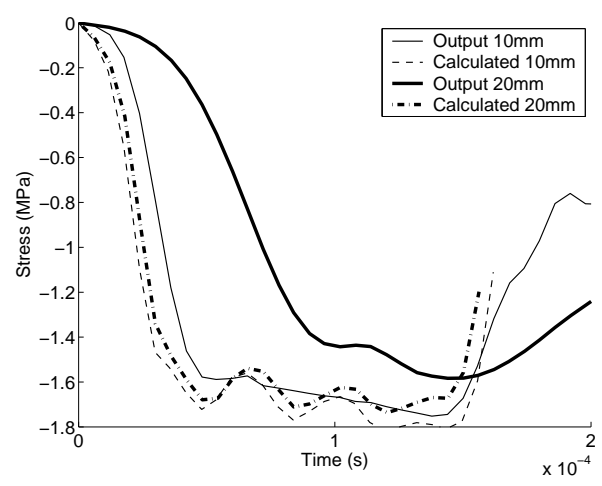

(b) Stress curves

Fig. 13. Comparison of the strain curves and stress curves obtained from the strain gauge and from the specimen directly (150 $\mathrm{mm}$ projectile). (a) Strain curves (b) Stress curves

\subsection{Simulation Results - FGFM specimen}

Once the FE model is quantitatively validated, it is possible to consider the wave propagation through a virtual FGFM. To illustrate the expected variation in stress/strain through the thickness due to the graded mechanical properties in this section, specimens of thickness $300 \mathrm{~mm}$ were adopted. This large thickness is still valid in the FEM simulations as the stress and strain are obtained directly from the simulation results, rather than calculated from the strain gauges.

The sensitivity of peak stress in each layer to the specimen thickness is shown in Fig. 15. The difference in peak values of stresses in different layers becomes more pronounced as the thickness increases. The magnitude of peak stress decreases with the increasing thickness as the stress wave is diminished more 


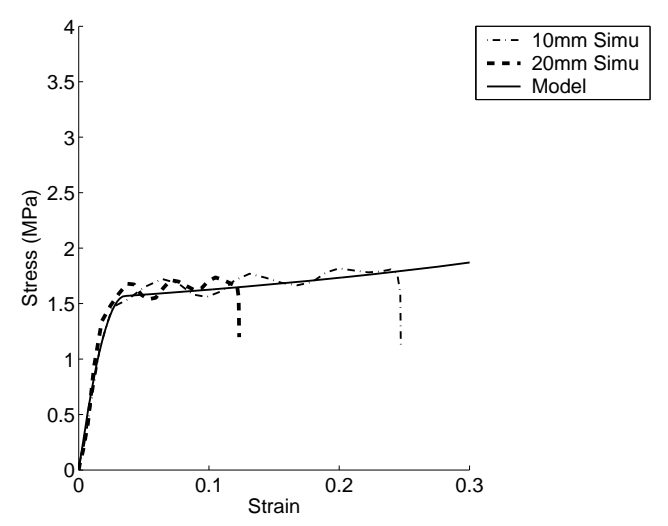

(a) $150 \mathrm{~mm}$ projectile

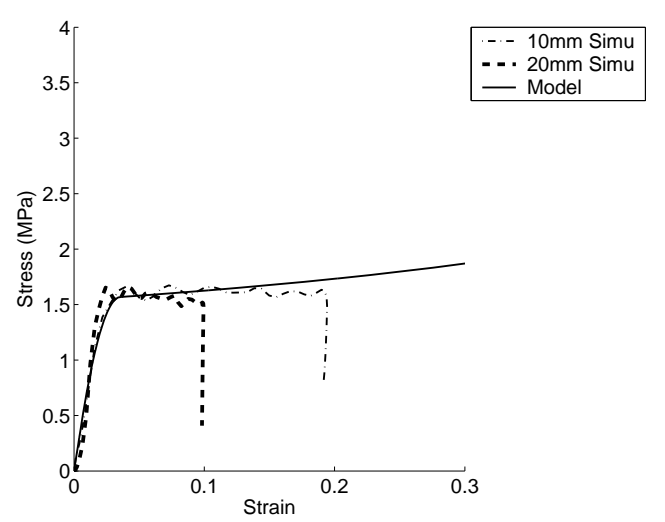

(b) $250 \mathrm{~mm}$ projectile

Fig. 14. The stress-strain curve obtained from the simulations. (a) $150 \mathrm{~mm}$ projectile (b) $250 \mathrm{~mm}$ projectile

through a thicker specimen. The evolution of stress in 10 selected layers in the uniform specimens of thickness 300 mm are illustrated in Fig. 16.

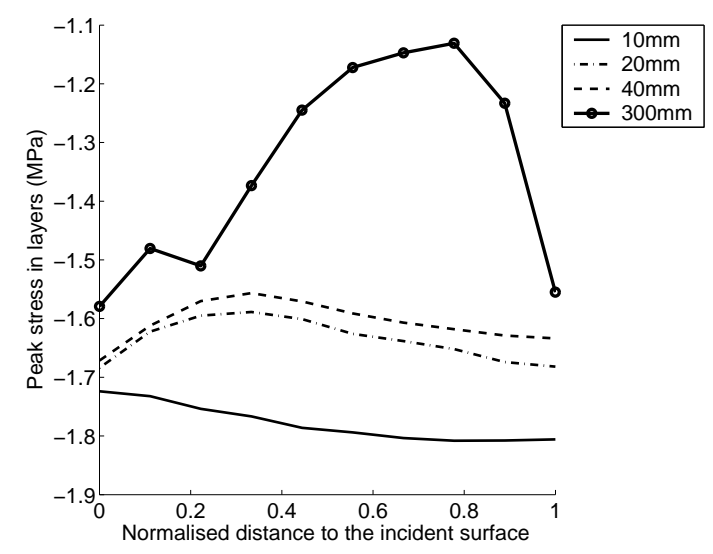

Fig. 15. Sensitivity of peak stress in layers to the specimen thickness.

The transmitted waves, as measured from the strain gauge on the transmitter bar for the uniform specimen and the FGFM specimens $\left(\Delta \rho=100 \mathrm{~kg} / \mathrm{m}^{3}\right)$ are illustrated in Fig. 17. The transmitted wave is obviously diminished more through the FGFM specimens with decreasing density. The graded specimens, with their density increasing according to the logarithmic and square root gradient functions, attenuate the wave more than the uniform specimen, while the graded specimens with their density increasing according to the other types of gradient functions (linear, quadratic, cubic) transmit more wave.

Following from the theory of elastic wave propagation, as a wave propagates from material 1 to material 2, the displacement amplitudes of the incident $\left(A_{i}\right)$, reflected $\left(A_{r}\right)$, and transmitted wave $\left(A_{t}\right)$ can be related by: 


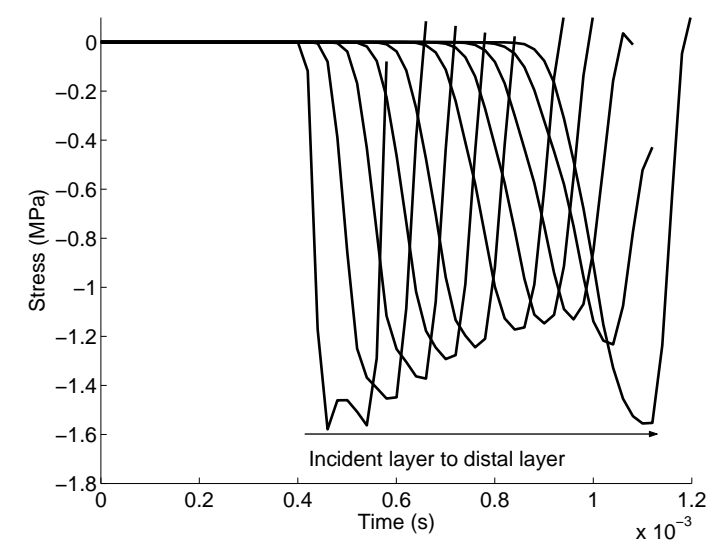

Fig. 16. Evolution of stress in 10 selected layers in the uniform specimen of thickness $300 \mathrm{~mm}$.

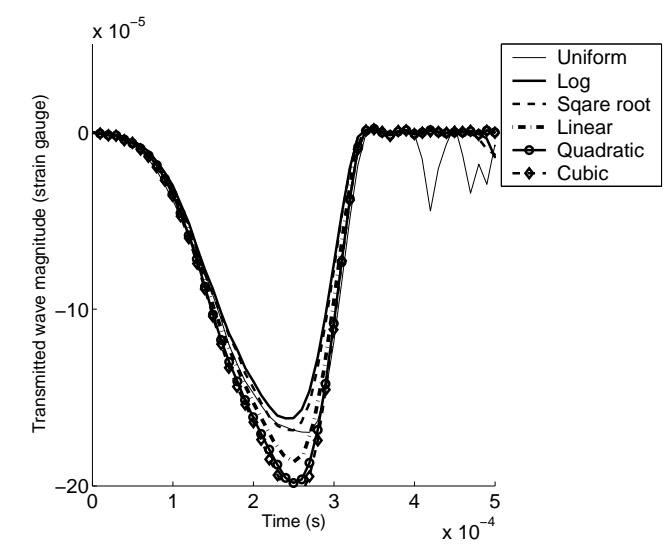

(a) Increasing density

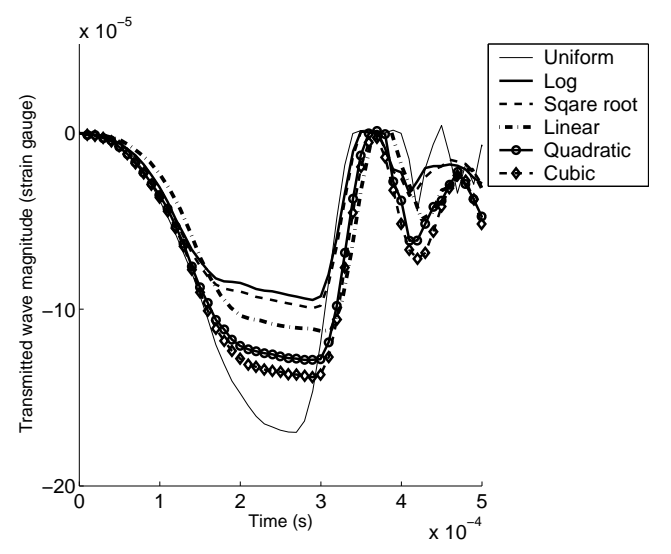

(b) Decreasing density

Fig. 17. Comparison of transmitted wave as measured from the strain gauge for the uniform specimen and the FGFM specimens $\left(\Delta \rho=100 \mathrm{~kg} / \mathrm{m}^{3}\right)$. (a) Increasing density (b) Decreasing density

$$
\begin{aligned}
A_{r} & =\frac{1-\alpha}{1+\alpha} A_{i} \\
A_{t} & =\frac{2}{1+\alpha} A_{i}
\end{aligned}
$$

where $\alpha$ is the impedance ratio defined as $\sqrt{\frac{\rho_{2} E_{2}}{\rho_{1} E_{1}}}, E$ is Young's Modulus, and $\rho$ is the density. The stress amplitudes of the incident $\left(\sigma_{i}\right)$, reflected $\left(\sigma_{r}\right)$, and transmitted wave $\left(\sigma_{t}\right)$ can be related as:

$$
\sigma_{r}=\frac{\alpha-1}{1+\alpha} \sigma_{i}
$$




$$
\sigma_{t}=\frac{2 \alpha}{1+\alpha} \sigma_{i}
$$

Equation (13) illustrates that, when an incident wave approaches a more compliant material (the impedance ratio is less than 1), the stress amplitude of the transmitted wave will be smaller than that of the incident wave; when an incident wave approaches a more stiff material (the impedance ratio is greater than 1), the stress amplitude of the transmitted wave will be greater than that of the incident wave.

As described above, the FGFM specimen is divided into 20 layers and so there are 19 graded interfaces where wave incidence and wave reflection occur. Although the FGFM specimens deform both elastically and plastically as the stress wave propagates, the ratio of transmitted stress in layer twenty to incident stress in layer one, accounting for just the FGFM's elastic properties, is calculated using Equation (13) simply for reference. Although there are multi-reflection and incidence at each interface, only the first transmission in each layer is considered for calculation as the following transmissions have time delays. These ratios, calculated from elastic wave theory, and the actual ratios predicted by simulation, which include plastic wave propagation, are compared in Table 3. It can be seen from Table 3 that, for the elastic wave, the ratio of stress magnitude for increasing density is highest in a logarithmic gradient, while it is lowest in a cubic gradient. As there is energy dissipation due to plastic deformation, the stress ratios are not the same as those from calculations, however, the trend in both cases agrees with what is expected, namely an increasing density gradient will amplify the resultant transmitted elastic stress, while a decreasing gradient will attenuate the transmitted elastic stress.

Table 3

Ratio of stress magnitude of wave in 20 th layer to that in 1st layer $\left(\Delta \rho=100 \mathrm{~kg} / \mathrm{m}^{3}\right)$

\begin{tabular}{|c|c|c|c|c|}
\hline \multirow{2}{*}{ Gradients } & \multicolumn{2}{|c|}{ Calculated ratio $(\mathrm{Eq}(13))$} & \multicolumn{2}{c|}{ Ratio in simulation } \\
\cline { 2 - 5 } & $\begin{array}{c}\text { Increasing } \\
\text { density }\end{array}$ & $\begin{array}{c}\text { Decreasing } \\
\text { density }\end{array}$ & $\begin{array}{c}\text { Increasing } \\
\text { density }\end{array}$ & $\begin{array}{c}\text { Decreasing } \\
\text { density }\end{array}$ \\
\hline Uniform & 1.000 & 1.000 & 0.985 & 0.985 \\
\hline Logarithmic & 1.396 & 0.896 & 1.538 & 0.445 \\
\hline Square Root & 1.380 & 0.895 & 1.589 & 0.464 \\
\hline Linear & 1.234 & 0.822 & 1.458 & 0.444 \\
\hline Quadratic & 1.171 & 0.800 & 1.503 & 0.461 \\
\hline Cubic & 1.146 & 0.792 & 1.389 & 0.471 \\
\hline
\end{tabular}

The peak stresses in layers in the specimen with various gradient functions $\left(\Delta \rho=100 \mathrm{~kg} / \mathrm{m}^{3}\right)$ are illustrated in Fig. 18. The stress is seen to be shaped 
by the functionally graded densities. By comparing Fig. 18 and Fig. 10, it can be seen that the shape of the peak stress is similar to the shape of the density gradients. It is expected that the magnitude of stress increases as the wave propagates to layers of higher density and decreases as the wave propagates to layers of lower density. However, this trend is not always true in the intermediate layers for the increasing densities. The global trend at the two free surfaces is true (confirmed from Table 3). This trend is more obvious in the specimens with decreasing densities. The magnitudes of stresses monotonically decrease for the logarithmic, square root, and linear gradient functions.

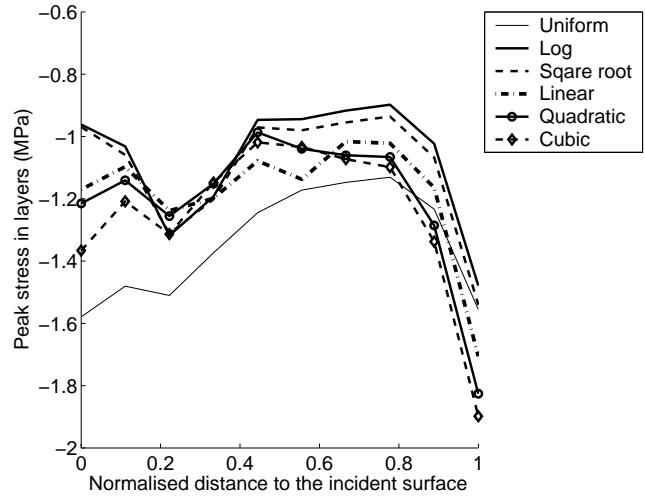

(a) Increasing density

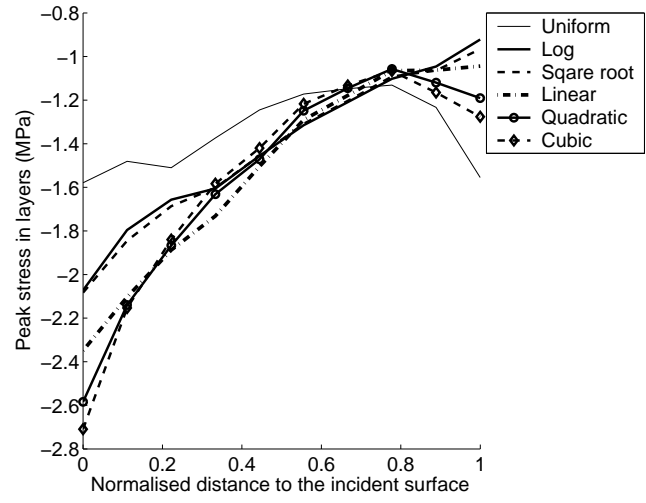

(b) Decreasing density

Fig. 18. Comparison of peak stresses in layers between various gradient functions $\left(\Delta \rho=100 \mathrm{~kg} / \mathrm{m}^{3}\right)$. (a) Increasing density (b) Decreasing density

As listed in Table 2, for the specimens with increasing densities, the densities at the incident surface increase as the gradient function changes from logarithmic to cubic: this is consistent with the magnitudes of stresses as shown in Fig. 18(a). As the wave propagates to the distal surface, the densities all increase to values higher than the uniform specimen. However, the magnitudes of stresses in the specimens with logarithmic and square root gradient functions are still lower than that in the uniform specimen. The comparison of magnitudes of stresses at the distal surface (Fig. 18(a)) agree with that of the transmitted wave as measured from the strain gauge on the transmitter bar (Fig. 17(a)). For the specimens with decreasing densities, the densities at the incident surface increase as the gradient function changes from logarithmic to cubic, which are consistent with the magnitudes of stresses as shown in Fig. 18(b). As the wave propagates to the distal surface, the densities all decrease to values lower than that of the uniform specimen and the magnitudes of stresses also decrease to values lower than that of the uniform specimen. The magnitudes of stresses at the distal surface also agree with the transmitted wave measured from the transmitter bar (Fig. 18(b)).

The sensitivity of the peak stress in layers to the density range $(\Delta \rho)$ is shown 
in Fig. 19 for the linear gradient. The difference in peak stress between the incident and the distal surfaces is amplified by increasing the density range. The lower or higher magnitudes of peak stresses at both free surfaces are all consistent with the lower or higher values of densities at those surfaces.

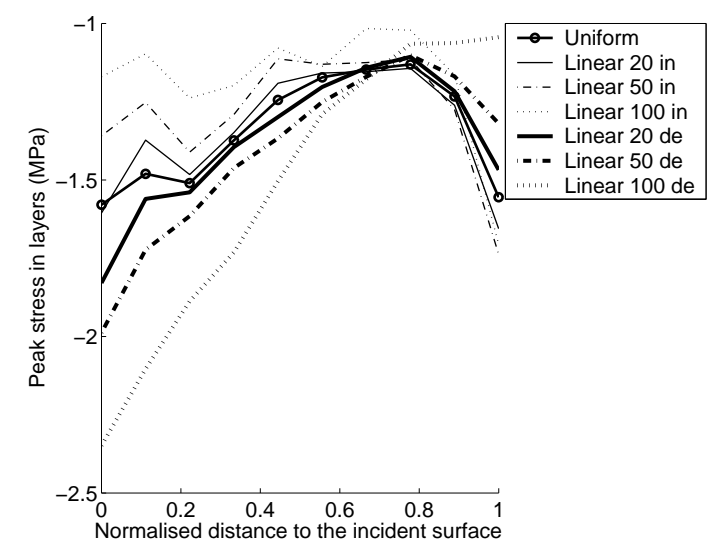

Fig. 19. Comparison of peak stresses in layers between various density ranges (linear gradient; in-increasing density; de-decreasing density.)

The peak plastic dissipation energy density in layers in the specimen with various gradient functions $\left(\Delta \rho=100 \mathrm{~kg} / \mathrm{m}^{3}\right)$ are illustrated in Fig. 20. The plastic dissipation energy density is the total energy dissipated per unit volume in the element by plastic deformation. It is clearly shown that most of the energy dissipates in the first layer at the incident surface. The layer at the distal surface also dissipates a large proportion of energy.

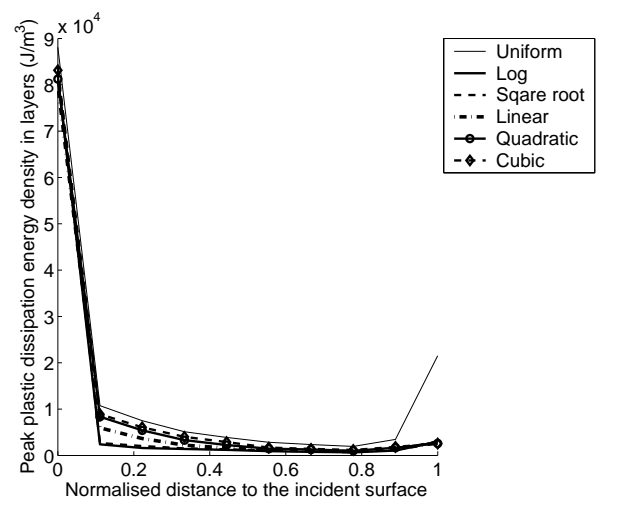

(a) Increasing density

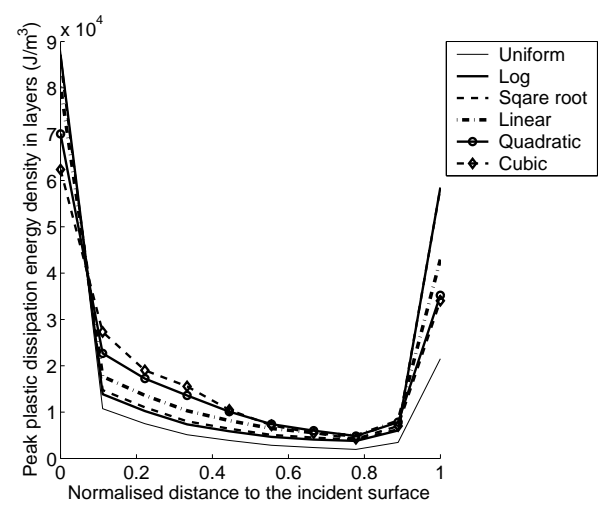

(b) Decreasing density

Fig. 20. Comparison of peak plastic dissipation energy density in layers between various gradient functions $\left(\Delta \rho=100 \mathrm{~kg} / \mathrm{m}^{3}\right)$. (a) Increasing density (b) Decreasing density

In the first layer at the incident surface for the increasing density, the density (and also the peak stress, Fig. 18) increases from logarithmic to cubic gradient 
functions and to uniform (Fig. 10), while the plastic dissipation energy density decreases from logarithmic to cubic gradient functions and to the uniform (Fig. 20(a)). This is because the yield stress also increases as the density increases, and fewer plastic strains occurs in the higher density layers even though the peak stresses occur. From the second layer, this trend reverses.

In the first layer at the incident surface for the decreasing density, the density (and also the peak stress, Fig. 18) decreases from cubic to logarithmic gradient functions and to uniform (Fig. 10), while the plastic dissipation energy density increases from cubic to logarithmic gradient functions and to the uniform (Fig. 20(b)). From the second layer, this trend also reverses. At the last layer at the distal surface, the trend alters again, i.e. the energy density in the uniform density exhibits the lowest value, and it increases from the cubic to logarithmic gradient functions.

The sensitivity of the peak plastic dissipation energy density in layers to the density range $(\Delta \rho)$ for linear gradient is shown in Fig. 21. The plastic dissipation energy density is highest for the widest density range with decreasing density range (100 de) and lowest for the widest density range with increasing density range (100 in). However, the density value and the magnitude of peak stress at the distal surface for the widest density range with decreasing density range $(100 \mathrm{de})$ is the lowest. The reason for this is that the lowest yield stress is associated with this density.

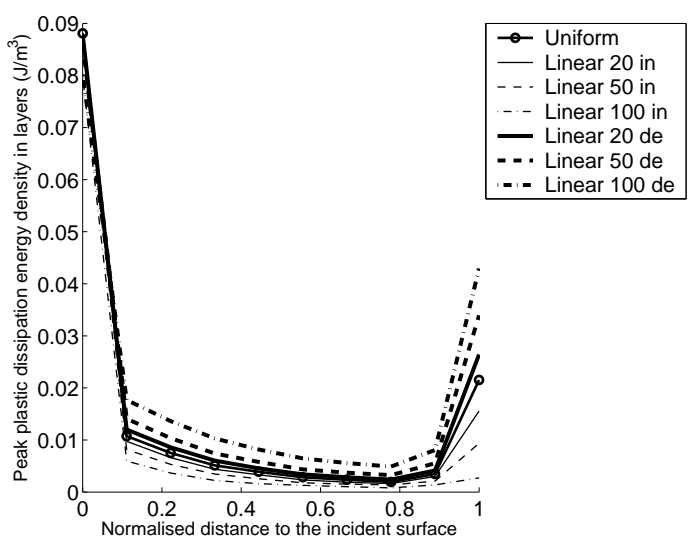

Fig. 21. Comparison of peak plastic dissipation energy density in layers between various density ranges (linear gradient; in-increasing density; de-decreasing density.)

The plastic mechanism associated with the fact that the FGFMs absorb more energy than the uniform foam can be explained as follows:

The main mechanism by which a foam absorbs energy / attenuates stress is through plastic deformation. As a stress wave propagates along the $z$-direction through a uniform foam, it will plastically deform the foam until its magnitude $\sigma_{w}$ becomes lower than the foam's yield stress $\sigma_{y}$. Once this occurs, the remainder of the wave will propagate elastically with little additional energy 
dissipated since no more plastic deformation will occur. It can be deduced that if $\sigma_{y}$ were to decrease, or $\sigma_{w}$ were to increase in the $z$-direction there would be a time delay in $\sigma_{w}$ attenuating to the value of $\sigma_{y}$, allowing greater amounts of energy to be absorbed plastically. This is exactly what an FGFM is designed to achieve: the yield stress $\sigma_{y}$ of a graded foam diminishes in the $z$-direction as the density decreases, or the wave stress $\sigma_{w}$ of a graded foam amplifies in the $z$-direction as the density increases. Ideally, in order to maximise the amount of plastic deformation in a graded foam, the yield stress $\sigma_{y}$ at any point $z$ should be equal to the stress wave magnitude $\sigma_{w}$ at $z$, while the change in yield stress between any two points $z$ and $z+\delta z$ should be greater than $\delta \sigma_{w}$ over the distance $\delta z$. Symbolically:

$$
\begin{aligned}
\left.\sigma_{y}\right|_{z} & =\left.\sigma_{w}\right|_{z}, \forall z \\
\frac{\partial \sigma_{y}}{\partial z} & \geq \frac{\partial \sigma_{w}}{\partial z}
\end{aligned}
$$

In essence, the plastic dissipation energy density is a combined result of the achieved stress level and yield stress. A comprehensive study of this can lead to an optimised density range and gradient function for each amplitude of incident wave. The work of Cui et al [13] provides a series of such results.

\section{Conclusions}

The current study established an FEM model of the SHPB test and validated it against a complementary set of laboratory SHPB tests. Wave propagation through the FGFMs were analysed using this FEM model. The principle findings of this study are outlined as follows:

- The plateau stress of ALPORAS ${ }^{\circledR}$ foam is shown to be strain rate sensitive when moving from quasi-static $(\ll 1 / \mathrm{s})$ to strain rates on the order of $1000 / \mathrm{s}$. However higher strain rates $(\approx 2000 / s)$ appeared to influence the plateau stress only slightly. This indicates that once dynamic conditions are reached, ALPORAS ${ }^{\circledR}$ foam becomes relatively strain rate insensitive. Sensitivity from static to dynamic conditions will be implemented into the present FEM model in due course.

- Corrections for wave dispersion should be accounted for when using low impedance pressure bars, i.e. a purely elastic behavior cannot be assumed. This can be achieved using Fourier Theory and knowledge of the materials viscoelastic behavior.

- The variation in a cellular FGFM would make it extremely difficult to dynamically test and obtain valid results using a traditional SHPB apparatus, 
due to the limiting assumptions that are made for Equations (1) to (3). Variables that a FGM would introduce, such as a spatially varying yield stress, varying density and/or varying strain rate sensitivities, would almost certainly invalidate any efforts in assuring stress-strain uniformity, in which case only an average stress-strain result could be obtained. This problem is overcome by virtually testing such materials since Equations (1) to (3) are not necessary to calculate the constitutive outputs.

- The amplitude of a stress wave propagating through a FGFM can be shaped by the gradient functions according to which the foam density varies through the direction of wave propagation.

- The local plastic dissipation energy density is a combined result of the achieved local stress level and the local yield stress. Optimum gradient function and density range can be achieved by the optimisation design using this FEM model.

The current study considered an FGFM graded in one dimension and has shown that improved energy management can be obtained under uni-axial loading conditions, however it would be unreasonable to assume that such a gradient would provide an advantage in impact management in any scenario other than if the direction of the impact coincides very closely to the direction of the gradient. Hypothetically speaking, an FGFM graded in three mutually orthogonal dimensions may be more suitable for general triaxial. However, the influence and interdependency that would inevitably arise from three mutually orthogonal gradients would complicate the problem description and hence the solving procedure many times over. It is likely that an FGFM would be most advantageous for applications in which the direction of a likely impact can be predicted with reasonable accuracy. For example, the force to a helmet during an impact can, in the majority of cases, be assumed to act approximately normal to the point of impact (assuming the horizontal component of the fall is small compared to the vertical component), i.e., through the thickness of a helmet liner, for which the principle direction of an impact is typically also through the thickness.

\section{Acknowledgements}

This study is funded by Enterprise Ireland (PC/2005/071) and the Turf Club of Ireland. 


\section{References}

[1] N. Hilyard, L. Djiauw, Observations on the impact behaviour of polyurethane foams; i. the polymer matrix, Journal of Cellular Plastics 7 (1971) $33-42$.

[2] N. Mills, A. Gilchrist, The effectiveness of foams in bicycle and motorcycle helmets, Accid. Anal. and Prev. 23 (1991) 153 - 163.

[3] L. D. Landro, G. Sala, D. Olivieri, Deformation mechanisms and energy absorption of polystyrene foams for protective helmets, Polymer Testing 21 (2002) $217-228$.

[4] V. Deshpande, N. Fleck, High strain rate compressive behaviour of aluminium alloy foams, International Journal of Impact Engineering 44 (2000) 277-298.

[5] J. L. Jr, K. A. Dannemann, Strain rate effects in porous materials, porous and cellular materials for structural applications, Materials Research Society Symposium Proceedings 521 (1998) 103-108.

[6] M. Peroni, L. Peroni, M. Avalle, High strain-rate compression test on metallic foam using a multiple pulse shpb apparatus, J. Phys. IV France 134 (2006) 609-616.

[7] H. Kanahashi, T. Mukai, Y. Yamada, K. Shimojima, M. Mabuchi, T. Nieh, K. Higashi, Dynamic compression of an ultra-low density aluminium foam, Material Science and Engineering A 280 (2000) 349-353.

[8] K. Dannemann, J. L. Jr, High strain rate compression of closed-cell aluminium foams, Material Science and Engineering A 293 (2000) 157-164.

[9] I. Elnasri, S. Pattofatto, H. Zhao, H. Tsitsiris, F. Hild, Y. Girard, Shock enhancement of cellular structures under impact loading: Part i experiments, Journal of the Mechanics and Physics of Solids 55 (2007) 2652 - 2671.

[10] H. Zhao, S. Abdennadher, On the strength enhancement under impact loading of square tubes made from rate insensitive metals, Int. J. Solids Structures 41.

[11] J. W. Klintworth, Dynamic crushing of cellular solids, Ph.D. thesis, University of Cambridge (1989).

[12] S. Reid, C. Peng, Dynamic uniaxial crushing of wood, International Journal Of Impact Engineering 19 (1997) 531-570.

[13] L. Cui, S. Kiernan, M. D. Gilchrist, Designing the energy absorption capacity of functionally graded foam materials, Materials Science and Engineering A In press.

[14] H. A. Bruck, A one-dimensional model for designing functionally graded materials to manage stress waves, International Journal of Solids and Structures 37 (2000) $6383-6395$. 
[15] A. Berezovski, J. Engelbrecht, G. A. Maugin, Numerical simulation of twodimensional wave propagation in functionally graded materials, European Journal of Mechanics A/Solids 22 (2003) 257-256.

[16] A. H. Brothers, D. C. Dunand, Mechanical properties of a density-graded replicated aluminium foam, Material Science and Engineering A 489 (2008) $439-443$.

[17] Y. Matsumoto, A. Brothers, S. Stock, D. Dunand, Uniform and graded chemical milling of aluminum foams, Materials Science and Engineering A 447 (2007) 150 -157 .

[18] B. Kieback, A. Neubrand, H. Riedel, Processing techniques for functionally graded materials, Materials Science and Engineering A 362 (2003) 81 - 105.

[19] AMSE, Split-hopkinson pressure bar apparatus. an historic mechanical engineering landmark, Designation Ceremony Southwest Research Institute.

[20] M. M. Al-Mousawi, S. R. Reid, W. F. Deans, The use of the split hopkinson pressure bar techniques in high strain rate materials testing, Proc Instn Mech Engrs Part C 211 (1997) 273 - 292.

[21] A. Bragov, A. Lomunov, I. Sergeichev, K. Tsembelis, W. Proud, Determination of physicomechanical properties of soft soils from medium to high strain rates, International Journal of Impact Engineering 35 (2008) 967 - 976.

[22] L. Ninan, J. Tsai, C. Sun, Use of split hopkinson pressure bar for testing off-axis composites, International Journal of Impact Engineering 25 (2001) 291 - 313.

[23] A. Sharma, A. Shukla, R. A. Prosser, Mechanical characterization of soft materials using high speed photography and split hopkinson pressure bar technique, Journal Of Materials Science 37 (2002) 1005 - 1017.

[24] O. Lee, M. Kim, Dynamic material property characterization by using split hopkinson pressure bar (shpb) technique, Nuclear Engineering and Design 226 (2004) $119-125$.

[25] W. Chen, B. Song, D. J. Frew, M. J. Forrestal, Dynamic small strain measurements of a metal specimen with a split hopkinson pressure bar, Society for Experimental Mechanics 43 (2003) 20 - 23.

[26] O. Sawas, N. S. Brar, R. A. Brockman, Dynamic characterization of compliant materials using an all-polymeric split hopkinson bar, ExperimentaI Mechanics 38 (1998) $204-210$.

[27] W. Chen, F. Lu, D. J. Frew, M. J. Forrestal, Dynamic compression testing of soft materials, Journal of Applied Mechanics 69 (2002) 214 - 223.

[28] W. Chen, F. Lu, N. Winfree, High-strain-rate compressive behavior of a rigid polyurethane foam with various densities, Experimental Mechanics 42 (2002) $65-73$. 
[29] H. Zhao, G. Gary, On the use of shpb techniques to determine the dynamic behaviour of materials in the range of small strains., Int. J. Solids Structures (1996) $3363-3375$.

[30] R. H. Blanc, Transient wave propagation methods for determining the viscoelastic properties of solids, Journal of Applied Mechanics 60 (1993) 763 -768 .

[31] H. Zhao, G. Gary, A three dimensional analytical solution of the longitudinal wave propagation in an infinite linear viscoelastic cylindrical bar. application to experimental techniques, Journal of The Mechanics and Physics of Solids 43 (1995) $1335-1348$.

[32] ABAQUS, ABAQUS Analysis User's Manual, Version 6.7, (CABAQUS, Inc. (2007).

[33] M. Schraad, F. Harlow, A stochastic constitutive model for disordered cellular materials: Finite-strain uni-axial compression, International Journal of Solids and Structures 43 (2006) 3542 - 3568. 